\title{
Applications of Multivalent Functions Associated with Generalized Fractional Integral Operator
}

\author{
Jae Ho Choi \\ Department of Mathematics Education, Daegu National University of Education, Daegu, South Korea \\ Email: choijh@dnue.ac.kr
}

Received August 29, 2012; revised October 29, 2012; accepted November 10, 2012

\begin{abstract}
By using a method based upon the Briot-Bouquet differential subordination, we investigate some subordination properties of the generalized fractional integral operator $\mathcal{J}_{0, z}^{\lambda, \mu, v}$ which was defined by Owa, Saigo and Srivastava [1]. Some interesting further consequences are also considered.
\end{abstract}

Keywords: Multivalent Functions; Subordination; Gaussian Hypergeometric Function; Fractional Integral Operator

\section{Introduction}

Let $\mathcal{A}_{n}(p)$ denote the class of functions $f(z)$ of the form

$$
f(z)=z^{p}+\sum_{k=n}^{\infty} a_{p+k} z^{p+k},(p, n \in \mathbb{N}:=\{1,2,3, \cdots\}),
$$

which are analytic in the open unit disk

$\mathbb{U}=\{z: z \in \mathbb{C}$ and $|z|<1\}$. Also let $f$ and $g$ be analytic in $\mathbb{U}$ with $f(0)=g(0)$. Then we say that $f$ is subordinate to $g$ in $\mathbb{U}$, written $f \prec g$ or $f(z) \prec g(z)$, if there exists the Schwarz function $w$, analytic in $\mathbb{U}$ such that $w(0)=0,|w(z)|<1$ and $f(z)=g(w(z))(z \in \mathbb{U})$. We also observe that

$$
f(z) \prec g(z) \text { in } \mathbb{U}
$$

if and only if

$$
f(0)=g(0) \text { and } f(\mathbb{U}) \subset g(\mathbb{U})
$$

whenever $g$ is univalent in $\mathbb{U}$.

Let $a, b$ and $c$ be complex numbers with $c \neq 0,-1,-2, \cdots$. Then the Gaussian/classical hypergeometric function ${ }_{2} F_{1}(a, b ; c ; z)$ is defined by

$$
{ }_{2} F_{1}(a, b ; c ; z)=\sum_{k=0}^{\infty} \frac{(a)_{k}(b)_{k}}{(c)_{k}} \frac{z^{k}}{k !},
$$

where $(\eta)_{k}$ is the Pochhammer symbol defined, in terms of the Gamma function, by

$$
(\eta)_{k}=\frac{\Gamma(\eta+k)}{\Gamma(\eta)}= \begin{cases}1, & (k=0) \\ \eta(\eta+1) \cdots(\eta+k-1), & (k \in \mathbb{N}) .\end{cases}
$$

The hypergeometric function ${ }_{2} F_{1}(a, b ; c ; z)$ is analytic in $\mathbb{U}$ and if $a$ or $b$ is a negative integer, then it reduces to a polynomial.

For each $A$ and $B$ such that $-1 \leq B<A \leq 1$, let us define the function

$$
h(A, B ; z)=\frac{1+A z}{1+B z},(z \in \mathbb{U}) .
$$

It is well known that $h(A, B ; z)$, for $-1 \leq B \leq 1$, is the conformal map of the unit disk onto the disk symmetrical respect to the real axis having the center $(1-A B) /\left(1-B^{2}\right)$ and the radius $(A-B) /\left(1-B^{2}\right)$. The boundary circle cuts the real axis at the points $(1-A) /(1-B)$ and $(1+A) /(1+B)$.

Many essentially equivalent definitions of fractional calculus have been given in the literature (cf., e.g. [2,3]). We state here the following definition due to Saigo [4] (see also $[1,5]$ ).

Definition 1. For $\lambda>0, \mu, v \in \mathbb{R}$, the fractional integral operator $\mathcal{I}_{0, z}^{\lambda, \mu, v}$ is defined by

$$
\begin{aligned}
& \mathcal{I}_{0, z}^{\lambda, \mu, v} f(z) \\
& =\frac{z^{-\lambda-\mu}}{\Gamma(\lambda)} \int_{0}^{z}(z-\zeta)_{2}^{\lambda-1} F_{1}\left(\lambda+\mu,-v ; \lambda ; 1-\frac{\zeta}{z}\right) f(\zeta) \mathrm{d} \zeta,
\end{aligned}
$$

where ${ }_{2} F_{1}$ is the Gaussian hypergeometric function defined by (1.2) and $f(z)$ is taken to be an analytic function in a simply-connected region of the z-plane containing the origin with the order

$$
f(z)=\mathcal{O}\left(|z|^{\epsilon}\right)(z \rightarrow 0)
$$

for $\epsilon>\max \{0, \mu-v\}-1$, and the multiplicity of $(z-\zeta)^{\lambda-1}$ is removed by requiring that $\log (z-\zeta)$ to be real when $z-\zeta>0$. 
The definition (1.5) is an interesting extension of both the Riemann-Liouville and Erdélyi-Kober fractional operators in terms of Gauss's hypergeometric functions.

With the aid of the above definition, Owa, Saigo and Srivastava [1] defined a modification of the fractional integral operator $\mathcal{J}_{0, z}^{\lambda, \mu, v}$ by

$$
\begin{aligned}
& \mathcal{J}_{0, z}^{\lambda, \mu, v} f(z) \\
& =\frac{\Gamma(p+1-\mu) \Gamma(\lambda+p+1+v)}{\Gamma(p+1) \Gamma(p+1-\mu+v)} z^{\mu} \mathcal{I}_{0, z}^{\lambda, \mu, v} f(z)
\end{aligned}
$$

for $f(z) \in \mathcal{A}_{n}(p)$ and $\mu-v-p<1$. Then it is observed that $\mathcal{J}_{0, z}^{\lambda, \mu, v}$ also maps $\mathcal{A}_{n}(p)$ onto itself as follows:

$$
\begin{aligned}
& \mathcal{J}_{0, z}^{\lambda, \mu, v} f(z) \\
& =z^{p}+\sum_{k=n}^{\infty} \frac{(p+1)_{k}(p+1-\mu+v)_{k}}{(p+1-\mu)_{k}(\lambda+p+1+v)_{k}} a_{p+k} z^{p+k} \\
& \left(\lambda>1 ; \mu-v-p<1 ; f \in \mathcal{A}_{n}(p)\right)
\end{aligned}
$$

We note that $\mathcal{J}_{0, z}^{\alpha, 0, \beta-1} f(z)=\mathcal{O}_{\beta}^{\alpha} f(z),(\alpha \geq 0 ; \beta>-1)$, where the operator $\mathcal{O}_{\beta}^{\alpha}$ was introduced and studied by Jung, Kim and Srivastava [6] (see also [7]).

It is easily verified from (1.7) that

$$
\begin{aligned}
& z\left(\mathcal{J}_{0, z}^{\lambda, \mu, v} f(z)\right)^{\prime} \\
& =(\lambda+v+p) \mathcal{J}_{0, z}^{\lambda-1, \mu, v} f(z)-(\lambda+v) \mathcal{J}_{0, z}^{\lambda, \mu, v} f(z) .
\end{aligned}
$$

The identity (1.8) plays an important and significant role in obtaining our results.

Recently, by using the general theory of differential subordination, several authors (see, e.g. [7-9]) considered some interesting properties of multivalent functions associated with various integral operators. In this manuscript, we shall derive some subordination properties of the fractional integral operator $\mathcal{J}_{0, z}^{\lambda, \mu, \nu}$ by using the technique of differential subordination.

\section{Main Results}

In order to establish our results, we shall need the following lemma due to Miller and Mocanu [10].
Lemma 1. Let $h(t)$ be analytic and convex univalent in $\mathbb{U}$ with $h(0)=1$, and let $g(z)=1+b_{n} z^{n}+b_{n+1} z^{n+1}+\cdots$ be analytic in $\mathbb{U}$. If

$$
g(z)+\frac{1}{C} z g^{\prime}(z) \prec h(z),
$$

then for $c \neq 0$ and $\operatorname{Re} c \geq 0$,

$$
g(z) \prec \frac{c}{n} z^{-c / n} \int_{0}^{z} t^{c / n-1} h(t) \mathrm{d} t .
$$

We begin by proving the following theorem.

Theorem 1. Let $-1 \leq B<A \leq 1, \lambda>1, \lambda+v>-p$, $\mu-v-p<1, \quad \mu-1<p$ and $0<\alpha<1$, and let

$$
\begin{gathered}
f(z)=z^{p}+\sum_{k=n}^{\infty} a_{p+k} z^{p+k} \in \mathcal{A}_{n}(p) \text {. Suppose that } \\
\sum_{k=n}^{\infty} c_{k}\left|a_{p+k}\right| \leq 1,
\end{gathered}
$$

where

$c_{k}=\frac{1-B}{A-B} \frac{[\lambda+p+v+k(1-\alpha)](p+1)_{k}(p+1-\mu+v)_{k}}{(\lambda+p+v)(p+1-\mu)_{k}(\lambda+p+1+v)_{k}}$

and $(\eta)_{k}$ is given by (1.3).

1) If $-1 \leq B<0$, then

$$
(1-\alpha) \frac{\mathcal{J}_{0, z}^{\lambda-1, \mu, v} f(z)}{z^{p}}+\alpha \frac{\mathcal{J}_{0, z}^{\lambda, \mu, v} f(z)}{z^{p}} \prec h(A, B ; z) .
$$

2) If $-1 \leq B<0$ and $\gamma \geq 1$, then

$$
\begin{aligned}
& \operatorname{Re}\left\{\left(\frac{\mathcal{J}_{0, z}^{\lambda, \mu, v} f(z)}{z^{p}}\right)^{1 / \gamma}\right\} \\
& >\left\{\frac{\lambda+v+p}{n(1-\alpha)} \int_{0}^{1} u^{\frac{\lambda+v+p}{n(1-\alpha)}-1}\left(\frac{1-A u}{1-B u}\right) d u\right\}^{1 / \gamma},(z \in U) .
\end{aligned}
$$

The result is sharp.

Proof. 1) If we set

$$
L=(1-\alpha) \frac{\mathcal{J}_{0, z}^{\lambda-1, \mu, v} f(z)}{z^{p}}+\alpha \frac{\mathcal{J}_{0, z}^{\lambda, \mu, v} f(z)}{z^{p}},
$$

then, from (1.7) we see that

$$
L=1+\sum_{k=n}^{\infty} \frac{[\lambda+p+v+k(1-\alpha)](p+1)_{k}(p+1-\mu+v)_{k}}{(\lambda+p+v)(p+1-\mu)_{k}(\lambda+p+1+v)_{k}} a_{p+k} z^{k} .
$$

For $-1 \leq B<0$ and $z \in \mathbb{U}$, it follows from (2.3) that

$$
\left|\frac{L-1}{A-B L}\right|=\left|\frac{\sum_{k=n}^{\infty} \frac{[\lambda+p+v+k(1-\alpha)](p+1)_{k}(p+1-\mu+v)_{k}}{(\lambda+p+v)(p+1-\mu)_{k}(\lambda+p+1+v)_{k}} a_{p+k} z^{k}}{A-B-B \sum_{k=n}^{\infty} \frac{[\lambda+p+v+k(1-\alpha)](p+1)_{k}(p+1-\mu+v)_{k}}{(\lambda+p+v)(p+1-\mu)_{k}(\lambda+p+1+v)_{k}} a_{p+k} z^{k}}\right| \leq \frac{\sum_{k=n}^{\infty} c_{k}\left|a_{p+k}\right|}{1-B+B \sum_{k=n}^{\infty} c_{k}\left|a_{p+k}\right|} \leq 1,
$$


which implies that

$$
(1-\alpha) \frac{\mathcal{J}_{0, z}^{\lambda-1, \mu, v} f(z)}{z^{p}}+\alpha \frac{\mathcal{J}_{0, z}^{\lambda, \mu, v} f(z)}{z^{p}} \prec h(A, B ; z) .
$$

2) Let

$$
g(z)=\frac{\mathcal{J}_{0, z}^{\lambda, \mu, v} f(z)}{z^{p}},\left(f \in \mathcal{A}_{n}(p)\right) .
$$

Then the function $g(z)=1+b_{n} z^{n}+b_{n+1} z^{n+1}+\cdots$ is analytic in $\mathbb{U}$. Using (1.8) and (2.9), we have

$$
\frac{\mathcal{J}_{0, z}^{\lambda-1, \mu, v} f(z)}{z^{p}}=g(z)+\frac{1}{\lambda+v+p} z g^{\prime}(z) .
$$

From (2.5), (2.9) and (2.10) we obtain

$$
\begin{aligned}
& (1-\alpha) \frac{\mathcal{J}_{0, z}^{\lambda-1, \mu, v} f(z)}{z^{p}}+\alpha \frac{\mathcal{J}_{0, z}^{\lambda, \mu, v} f(z)}{z^{p}} \\
& =g(z)+\frac{1-\alpha}{\lambda+v+p} z g^{\prime}(z) \prec h(A, B ; z) .
\end{aligned}
$$

Thus, by applying Lemma 1, we observe that

$$
g(z) \prec \frac{\lambda+v+p}{n(1-\alpha)} z^{-\frac{\lambda+v+p}{n(1-\alpha)} z} \int_{0}^{\frac{\lambda+v+p}{n(1-\alpha)}-1}\left(\frac{1+A t}{1+B t}\right) \mathrm{d} t
$$

or

$$
\frac{\mathcal{J}_{0, z}^{\lambda, \mu, v} f(z)}{z^{p}}=\frac{\lambda+v+p}{n(1-\alpha)} \int_{0}^{1} u^{\frac{\lambda+v+p}{n(1-\alpha)}-1}\left(\frac{1+A u w(z)}{1+\operatorname{Buw}(z)}\right) \mathrm{d} u,(2
$$

where $w(z)$ is analytic in $\mathbb{U}$ with $w(0)=0$ and $|w(z)|<1(z \in \mathbb{U})$. In view of $-1 \leq B<A \leq 1$ and $\lambda+v>-p$, we conclude from (2.11) that

$\operatorname{Re}\left\{\frac{\mathcal{J}_{0, z}^{\lambda, \mu, v} f(z)}{z^{p}}\right\}>\frac{\lambda+v+p}{n(1-\alpha)} \int_{0}^{1} u^{\frac{\lambda+v+p}{n(1-\alpha)}-1}\left(\frac{1-A u}{1-B u}\right) \mathrm{d} u$, $(z \in \mathbb{U})$.

Since $\operatorname{Re}\left(w^{1 / \gamma}\right) \geq(\operatorname{Re} w)^{1 / \gamma}$ for $\operatorname{Re} w>0$ and $\gamma \geq 1$, from (2.12) we see that the inequality (2.6) holds.

To prove sharpness, we take $f(z) \in \mathcal{A}_{n}(p)$ defined by

$$
\frac{\mathcal{J}_{0, z}^{\lambda, \mu, v} f(z)}{z^{p}}=\frac{\lambda+v+p}{n(1-\alpha)} \int_{0}^{1} u^{\frac{\lambda+v+p}{n(1-\alpha)}-1}\left(\frac{1+A u z^{n}}{1+B u z^{n}}\right) \mathrm{d} u .
$$

For this function we find that

and

$$
(1-\alpha) \frac{\mathcal{J}_{0, z}^{\lambda-1, \mu, v} f(z)}{z^{p}}+\alpha \frac{\mathcal{J}_{0, z}^{\lambda, \mu, v} f(z)}{z^{p}}=\frac{1+A z^{n}}{1+B z^{n}}
$$

$\frac{\mathcal{J}_{0, z}^{\lambda, \mu, v} f(z)}{z^{p}} \rightarrow \frac{\lambda+v+p}{n(1-\alpha)} \int_{0}^{1} u^{\frac{\lambda+v+p}{n(1-\alpha)}-1} \frac{1-A u}{1-B u} \mathrm{~d} u$ as $z \rightarrow \mathrm{e}^{i \pi / n}$.

Hence the proof of Theorem 1 is evidently completed.
Theorem 2. Let $-1 \leq B<A \leq 1, \lambda>1, \lambda+v>-p$, $\mu-v-p<1, \mu-1<p$ and $0<\alpha<1$. Suppose that $f(z)=z^{p}+\sum_{k=n}^{\infty} a_{p+k} z^{p+k} \in \mathcal{A}_{n}(p), s_{1}(z)=z^{p}$ and $S_{m}(z)=z^{p}+\sum_{k=n}^{n+m-2} a_{p+k} z^{p+k}(m \geq 2)$. If the sequence $\left\{c_{k}\right\}$ is nondecreasing with

$$
c_{k} \geq \frac{(1-B)[\lambda+p+v+k(1-\alpha)]}{(A-B)(\lambda+p+v)}(k \geq n),
$$

where $c_{k}$ is given by (2.4) and satisfies the condition (2.3), then

$$
\operatorname{Re}\left\{\frac{\mathcal{J}_{0, z}^{\lambda, \mu, v} f(z)}{s_{m}(z)}\right\}>0
$$

and

$$
\operatorname{Re}\left\{\frac{s_{m}(z)}{\mathcal{J}_{0, z}^{\lambda, \mu, v} f(z)}\right\}>0
$$

Each of the bounds in (2.14) and (2.15) is best possible for $m \in \mathbb{N}$.

Proof. We prove the bound in (2.14). The bound in (2.15) is immediately obtained from (2.14) and will be omitted. Let

$$
h(z)=\frac{\mathcal{J}_{0, z}^{\lambda, \mu} f(z)}{s_{m}(z)}\left(f \in \mathcal{A}_{n}(p) ; z \in \mathbb{U}\right) .
$$

Then, from (1.7) we observe that

$$
h(z)=1+\frac{\sum_{k=n}^{n+m-2}\left(\delta_{k}-1\right) a_{p+k} z^{k}+\sum_{k=n+m-1}^{\infty} \delta_{k} a_{p+k} z^{k}}{1+\sum_{k=n}^{n+m-2} a_{p+k} z^{k}},
$$

where, for convenience,

$$
\delta_{k}=\frac{(p+1)_{k}(p+1-\mu+v)_{k}}{(p+1-\mu)_{k}(\lambda+p+1+v)_{k}} .
$$

It is easily seen from (2.4) and (2.13) that $c_{k}>1$ and

$$
\delta_{k}=\frac{(A-B)(\lambda+p+v)}{(1-B)[\lambda+p+v+k(1-\alpha)]} c_{k} \geq 1 \text {. }
$$

Hence, by applying (2.3) and (2.16), we have

$$
\left|\frac{h(z)-1}{h(z)+1}\right|=\left|\frac{\sum_{k=n}^{n+m-2}\left(\delta_{k}-1\right) a_{p+k} z^{k}+\sum_{k=n+m-1}^{\infty} \delta_{k} a_{p+k} z^{k}}{2+\sum_{k=n}^{n+m-2}\left(\delta_{k}+1\right) a_{p+k} z^{k}+\sum_{k=n+m-1}^{\infty} \delta_{k} a_{p+k} z^{k}}\right|
$$

$$
\leq \frac{\sum_{k=n}^{n+m-2}\left(\delta_{k}-1\right)\left|a_{p+k}\right|+\sum_{k=n+m-1}^{\infty} \delta_{k}\left|a_{p+k}\right|}{2-\sum_{k=n}^{n+m-2}\left(\delta_{k}+1\right)\left|a_{p+k}\right|-\sum_{k=n+m-1}^{\infty} \delta_{k}\left|a_{p+k}\right|} \leq 1(z \in \mathbb{U})
$$


which readily yields the inequality (2.14).

If we take $f(z)=z^{p}-z^{p+n+m-1}$, then

$$
\frac{f(z)}{s_{m}(z)}=1-z^{n+m-1} \rightarrow 0 \text { as } z \rightarrow 1^{-} \text {. }
$$

This show that the bound in (2.14) is best possible for each $m$, which proves Theorem 2 .

Finally, we consider the generalized Bernardi-LiveraLivingston integral operator $\mathcal{L}_{\sigma}(\sigma>-p)$ defined by (cf. [11-13])

$$
\mathcal{L}_{\sigma}(f)(z):=\frac{\sigma+p}{z^{\sigma}} \int_{0}^{z} t^{\sigma-1} f(t) \mathrm{d} t\left(f \in \mathcal{A}_{n}(p) ; \sigma>-p\right) .
$$

Theorem 3. Let $-1 \leq B<A \leq 1, \quad \sigma>-p, \lambda>1$, $\lambda+v>-p, \quad \mu-v-p<1, \quad \mu-1<p$ and $0<\alpha<1$, and let $f(z)=z^{p}+\sum_{k=n}^{\infty} a_{p+k} z^{p+k} \in \mathcal{A}_{n}(p)$. Suppose that

$$
\sum_{k=n}^{\infty} d_{k}\left|a_{p+k}\right| \leq 1
$$

where

$$
d_{k}=\frac{1-B}{A-B} \frac{[\sigma+p+k(1-\alpha)](p+1)_{k}(p+1-\mu+v)_{k}}{(\sigma+p+k)(p+1-\mu)_{k}(\lambda+p+1+v)_{k}}
$$

and $(\eta)_{k}$ is given by (1.3).

1) If $-1 \leq B<0$, then

$$
(1-\alpha) \frac{\mathcal{J}_{0, z}^{\lambda, \mu, v} f(z)}{z^{p}}+\alpha \frac{\mathcal{J}_{0, z}^{\lambda, \mu, v} \mathcal{L}_{\sigma}(f)(z)}{z^{p}} \prec h(A, B ; z) .
$$

2) If $-1 \leq B<0$ and $\gamma \geq 1$, then

$$
\begin{aligned}
& \operatorname{Re}\left\{\left(\frac{\mathcal{J}_{0, z}^{\lambda, \mu, v} \mathcal{L}_{\sigma}(f)(z)}{z^{p}}\right)^{1 / \gamma}\right\} \\
& >\left\{\frac{\sigma+p}{n(1-\alpha)} \int_{0}^{1} u^{\frac{\sigma+p}{n(1-\alpha)}-1}\left(\frac{1-A u}{1-B u}\right) \mathrm{d} u\right\}^{1 / \gamma}(z \in U) .
\end{aligned}
$$

The result is sharp.

Proof. 1) If we put

$$
M=(1-\alpha) \frac{\mathcal{J}_{0, z}^{\lambda, \mu, v} f(z)}{z^{p}}+\alpha \frac{\mathcal{J}_{0, z}^{\lambda, \mu, v} \mathcal{L}_{\sigma}(f)(z)}{z^{p}},
$$

then, from (1.7) and (2.17) we have

$$
\begin{aligned}
M= & 1+\sum_{k=n}^{\infty} \frac{[\sigma+p+k(1-\alpha)](p+1)_{k}(p+1-\mu+v)_{k}}{(\sigma+p+k)(p+1-\mu)_{k}(\lambda+p+1+v)_{k}} \\
& \cdot a_{p+k} z^{k} .
\end{aligned}
$$

Therefore, by using same techniques as in the proof of Theorem 1 1), we obtain the desired result.
2) From (2.17) we have

$$
\begin{aligned}
& (\sigma+p) \mathcal{J}_{0, z}^{\lambda, \mu, v} f(z) \\
& =\sigma \mathcal{J}_{0, z}^{\lambda, \mu, v} \mathcal{L}_{\sigma}(f)(z)+z\left(\mathcal{J}_{0, z}^{\lambda, \mu, v} \mathcal{L}_{\sigma}(f)(z)\right)^{\prime} .
\end{aligned}
$$

Let

$$
g(z)=\frac{\mathcal{J}_{0, z}^{\lambda, \mu, v} \mathcal{L}_{\sigma}(f)(z)}{z^{p}}(z \in \mathbb{U}) .
$$

Then, by virtue of (2.21), (2.22) and (2.19), we observe that

$$
\begin{aligned}
& (1-\gamma) \frac{\mathcal{J}_{0, z}^{\lambda, \mu, v} f(z)}{z^{p}}+\gamma \frac{\mathcal{J}_{0, z}^{\lambda, \mu, v} \mathcal{L}_{\sigma}(f)(z)}{z^{p}} \\
& =g(z)+\frac{1-\gamma}{\sigma+p} z g^{\prime}(z) \prec h(A, B ; z) .
\end{aligned}
$$

Hence, by applying the same argument as in the proof of Theorem 12 ), we obtain (2.20), which evidently proves Theorem 3.

\section{Acknowledgements}

This work was supported by Daegu National University of Education Research grant in 2011.

\section{REFERENCES}

[1] S. Owa, M. Saigo and H. M. Srivastava, "Some Characterization Theorems for Starlike and Convex Functions Involving a Certain Fractional Integral Operator," Journal of Mathematical Analysis and Applications, Vol. 140, No. 2, 1989, pp. 419-426. doi:10.1016/0022-247X(89)90075-9

[2] S. G. Samko, A. A. Kilbas and O. I. Marichev, "Fractional Integral and Derivatives, Theory and Applications," Gordon and Breach, New York, Philadelphia, London, Paris, Montreux, Toronto, Melbourne, 1993.

[3] H. M. Srivastava and R. G. Buschman, "Theory and Applications of Convolution Integral Equations," Kluwer Academic Publishers, Dordrecht, Boston, London, 1992.

[4] M. Saigo, "A Remark on Integral Operators Involving the Gauss Hypergeometric Functions," Mathematical Reports, Kyushu University, Vol. 11, No. 2, 1977-1978, pp. 135143.

[5] J. H. Choi, "Note on Differential Subordination Associated with Fractional Integral Operator," Far East Journal of Mathematical Sciences, Vol. 26, No. 2, 2007, pp. 499511.

[6] I. B. Jung, Y. C. Kim and H. M. srivastava, "The Hardy Space of Analytic Functions Associated with Certain OneParameter Families of Integral Operators," Journal of Mathematical Analysis and Applications, Vol. 176, No. 1, 1993, pp. 138-147. doi:10.1006/jmaa.1993.1204

[7] J.-L. Liu, "Notes on Jung-Kim-Srivastava Integral Operator," Journal of Mathematical Analysis and Applications, Vol. 294, No. 1, 2004, pp. 96-103. doi:10.1016/i.jmaa.2004.01.040 
[8] R. M. EL-Ashwash and M. K. Aouf, "Some Subclasses of Multivalent Functions Involving the Extended Fractional Differintegral Operator," Journal of Mathematical Inequalities, Vol. 4, No. 1, 2010, pp. 77-93.

[9] J. Patel, A. K. Mishra and H. M. Srivastava, "Classes of Multinalent Analytic Functions Involving the DziokSrivastava Operator," Computers and Mathematics with Applications, Vol. 54, No. 5, 2007, pp. 599-616. doi:10.1016/j.camwa.2006.08.041

[10] S. S. Miller and P. T. Mocanu, "Differential Subordinations and Univalent Functions," Michigan Mathematical Journal, Vol. 28, No. 2, 1981, pp. 157-172. doi: $10.1307 / \mathrm{mmj} / 1029002507$
[11] S. D. Bernardi, "Convex and Starlike Univalent Functions," Transactions of the American Mathematical Society, Vol. 135, 1969, pp. 429-446. doi:10.1090/S0002-9947-1969-0232920-2

[12] R. J. Libera, "Some Classes of Regular Univalent Functions," Proceedings of the American Mathematical Society, Vol. 16, No. 4, 1965, pp. 755-758. doi:10.1090/S0002-9939-1965-0178131-2

[13] H. M. Srivastava and S. Owa, Eds., "Current Topics in Analytic Function Theory," World Scientific Publishing Company, Singapore, New Jersey, London, Hong Kong, 1992. doi: $10.1142 / 1628$ 